\title{
An observational study using ultrasound to assess physiological changes following fluid bolus administration in paediatric sepsis in the emergency department
}

Elliot Long ${ }^{1,2,3^{*}}$ (D) Ed Oakley ${ }^{1,2,3}$, Franz E. Babl ${ }^{1,2,3}$, Trevor Duke $2,3,4$ and Paediatric Research in Emergency

Departments International Collaborative (PREDICT)

\begin{abstract}
Background: Fluid bolus administration is widely recommended as part of the initial treatment of paediatric sepsis, though the physiological benefits and harms are unclear. The primary aim of this study is to determine the effect of fluid bolus administration on cardiac index $(\mathrm{Cl})$. Secondary aims are to determine the effect of fluid bolus administration on extra-vascular lung water (EVLW), whether fluid responsiveness can be predicted by inferior vena cava (IVC) collapsibility, and whether fluid responsiveness correlates with changes in vital signs.
\end{abstract}

Methods/design: A prospective observational study of children presenting to the Emergency Department of The Royal Children's Hospital with clinically diagnosed sepsis requiring fluid bolus administration. Prior to fluid bolus administration, an echocardiogram, lung ultrasound, and IVC ultrasound will be performed, and vital signs recorded. These will be repeated $5 \mathrm{~min}$ after and $60 \mathrm{~min}$ after fluid bolus administration. Recorded echocardiograms and lung/IVC ultrasound will be evaluated independently by a paediatric cardiologist and paediatric emergency physician, respectively, blinded to the patient identity and time of examination relative to time of fluid bolus administration. Fifty patients will be enrolled in the study based on a precision based sample size calculation. Results will be analysed for change in $\mathrm{Cl}$ and change in EVLW 5 min after and 60 min after fluid bolus administration compared to baseline, IVC collapsibility as a predictor of fluid responsiveness, and the relationship between fluid responsiveness and changes in vital signs.

Discussion: This study will explore assumptions about the effect of fluid boluses on Cl in children with sepsis, and will provide evidence for secondary effects on other organ systems. This may lead to novel methods for assessment and decision making in the initial resuscitation of paediatric sepsis in clinical and research settings, and will likely influence the design of future interventional studies in this arena.

Trial registration: The study is registered with the Australian and New Zealand Clinical Trials Registry (ACTRN12614000824662; 04 August 2014).

Keywords: Sepsis, Fluid therapy, Ultrasound, Cardiac index, Extravascular lung water, Paediatric

\footnotetext{
* Correspondence: elliot.long@rch.org.au

'Department of Emergency Medicine, The Royal Children's Hospital, 50

Flemington Road, 3052 Parkville, VIC, Australia

${ }^{2}$ Murdoch Children's Research Institute, 50 Flemington Road, Parkville,

Australia

Full list of author information is available at the end of the article
} 


\section{Background}

Sepsis is the final common pathway and mode of death for many paediatric infectious processes, globally accounting for over 6 million childhood deaths per year, mostly in underdeveloped countries [1]. Case fatality rates are $5-10 \%$ in industrialised countries, and $20-30 \%$ in low and middle income countries [2, 3]. Despite being common and serious, the therapies for the initial resuscitation of paediatric sepsis are based on little or no evidence. In particular, fluid resuscitation therapy (FRT) is widely recommended for initial sepsis resuscitation $[4,5]$, but there is limited understanding of its potential beneficial and harmful effects, how the balance of benefits and harms may be influenced by patient and disease factors, and how this balance may be incorporated into treatment guidelines for translation into clinical use.

Fluid resuscitation was initially described in patients with severe dehydration during cholera epidemics in the early $19^{\text {th }}$ century [6], and subsequently applied to trauma patients with haemorrhagic shock [7]. Both of these disease states are characterised by intravascular volume depletion, and replacement of this intravascular volume with the same type of fluid that was lost helps to restore homeostasis. Fluid resuscitation for sepsis was popularised in 2001, when early goal-directed therapy (EGDT) was shown to improve survival in adults with septic shock when instituted in the emergency department (ED) [8]. This approach involved aggressive fluid resuscitation to achieve threshold central venous pressure (CVP) values, followed by early initiation of vasoactive agents for ongoing hypotension, and transfusion of blood for low central venous oxygen saturations $(\mathrm{ScvO} 2)$. As the initial therapy in EGDT, aggressive fluid resuscitation was the most commonly applied in practice, and the foundation upon which subsequent therapies were based. Since the publication of the EGDT protocol, the harms from aggressive fluid resuscitation and cumulative positive fluid balance have become apparent [9-16], and the benefit of EGDT compared to standard care has been called into question [17-19]. In particular, the Fluid Expansion As Supportive Therapy (FEAST) study found that FRT in Sub-Saharan African children with fever and signs of poor perfusion increased mortality compared to no FRT [20]. This raises serious questions about the role of FRT in the management of sepsis, and our understanding of the pathophysiological mechanisms underlying FRT in conditions where no volume loss has occurred.

Aggressive FRT with the aim of increasing stroke volume, with the assumption that this will improve end-organ perfusion and cellular oxygen use, may be physiologically flawed [21]. FRT into high-capacitance veins may in fact increase cardiac filling pressures faster than venous pressure due to limited diastolic compliance of the heart, thus reducing the pressure gradient driving venous return [22-24]. Increased venous pressure following FRT may reduce the gradient driving vital organ perfusion (MAP-CVP) [25]. Microcirculatory dysfunction through damage to the endothelial glycocalyx may be mediated partly by natriuretic peptides released as cardiac filling pressures increase following FRT [26-30]. The net effect of FRT in sepsis may be that it contributes significantly to end-organ oedema and dysfunction, and the progression from SIRS to multi-organ dysfunction and death $[9,10,12,13,31,32]$.

Limited published literature on the effect of FRT on cardiac output (CO) suggests that any effect is modest and transient. In a study of 20 adults with circulatory shock, the mean increase in $\mathrm{CO}$ immediately following FRT was $16 \%$, which decreased to $6 \%$ above baseline after sixty minutes [33]. In a separate study, 32 adults with septic shock were found to have a mean increase in CO of $15 \%$ immediately after FRT, which returned to $3 \%$ above baseline $40 \mathrm{~min}$ after FRT $(p<0.001)$ [34]. There is no published literature on the effect of FRT on cardiac index $(\mathrm{CI}=\mathrm{CO} /$ body surface area $(\mathrm{BSA}))$ in children. The ability to predict the response to FRT allows the clinician to restrict this therapy only to those that are fluid responsive. Fluid responsiveness is considered an increase in cardiac output of $10-15 \%$ in response to a fluid challenge (250-500 $\mathrm{ml}$ in adults) [35]. In the undifferentiated adult ICU population, as few as $50 \%$ of patients may be fluid responsive [35]. The remainder receive all of the potential harms from FRT with none of the potential benefits. IVC ultrasound is a dynamic method for assessing fluid responsiveness using cardiopulmonary interactions [36]. In spontaneously ventilating adults, IVC collapsibility of $>40 \%$ has been shown to be predictive of fluid responsiveness, while IVC collapsibility of $<15 \%$ is predictive of being fluid unresponsive [37]. No study has validated IVC collapsibility as a predictor of fluid responsiveness in children.

We set out to determine the effect of FRT on CI $5 \mathrm{~min}$ after and $60 \mathrm{~min}$ after fluid bolus administration in paediatric sepsis. Secondary aims include: the effect of FRT on extra-vascular lung water (EVLW), whether fluid responsiveness can be predicted by inferior vena cava (IVC) collapsibility, and whether fluid responsiveness correlates with changes in vital signs.

\section{Methods/design}

\section{Study design and setting}

The study is designed as a prospective observational study of children with sepsis. It will be conducted at a single paediatric ED in The Royal Children's Hospital ( $\mathrm{RCH})$, Melbourne, Australia. RCH is a tertiary children's hospital with an annual ED census of $>85,000$ children less than 18 years of age. 


\section{Inclusion and exclusion criteria}

Inclusion and exclusion are listed in Table 1. Inclusion criteria are based on international consensus definitions for the diagnosis of sepsis in children [38].

\section{Study procedure}

Patients who fulfil the eligibility criteria and whose parents provide written informed consent will be entered into the study. Prior to administration of a standardised fluid bolus, transthoracic echocardiography, lung and IVC ultrasound will be performed, and vital signs recorded (Fig. 1). Five minutes and $60 \mathrm{~min}$ after administration of a standardised fluid bolus, the same measurements will be taken. All ultrasound measurements will be taken by the Principal Investigator (PI), who has a Post Graduate Certificate in Echocardiography through The University of Melbourne, Australia. Recorded echocardiograms will subsequently be assessed by a blinded paediatric cardiologist. Lung ultrasound and IVC ultrasound will be assessed by a blinded paediatric emergency physician with a Diploma in Diagnostic Ultrasound (Australian Society for Ultrasound in Medicine). Vital signs that will be recorded from the patients monitor (Phillips MP70, Phillips Healthcare, Andover, MA) include: heart rate $(\mathrm{HR})$, respiratory rate $(\mathrm{RR})$, blood pressure $(\mathrm{BP})$, and peripheral oxyhaemoglobin saturation ( $\mathrm{SpO} 2)$. Additionally, patient temperature in degrees celcius will be recorded and a $10 \mathrm{~s}$ video recording of the patient will be taken at each time point and used to assess conscious state, capillary refill, and respiratory effort. These video recordings will be scored in terms of Glasgow Coma Scale for conscious state, time in seconds for capillary refill, and Silverman Score for respiratory effort [39] by a blinded paediatric emergency physician. Final outcomes will be obtained from the patient medical record following discharge or death.

Table 1 Inclusion and exclusion criteria

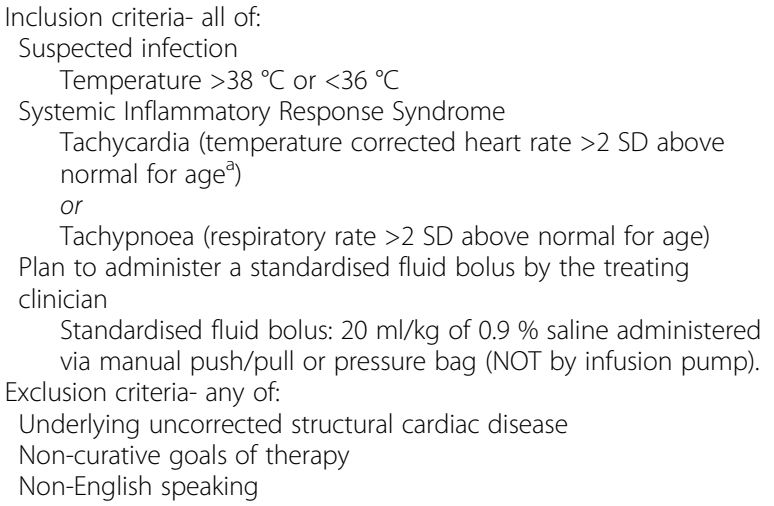

aheart rate was corrected by 10 beats per minute for every degree celcius above $38^{\circ}[55]$

\section{Study flow chart}

\section{Outcome measures}

The primary outcome measure is the percentage change in CI 5 and 60 min after standardised fluid bolus administration compared to baseline.

Secondary outcome measures include the change in EVLW after standardised fluid bolus administration, whether IVC collapsibility is predictive of fluid responsiveness, and whether the observed change in cardiac output following standardised fluid bolus administration is correlated with changes in vital signs and clinical observations. We also collected the following outcome data: ICU admission, requirement for inotrope/vasopressor infusion, requirement for non-invasive or invasive ventilation, requirement for renal replacement therapy, requirement for extracorporeal membrane oxygenation (ECMO), final microbiological diagnosis, and mortality.

\section{Ultrasound techniques}

Cardiac index will be measured by trans-thoracic echocardiography using a Zonare Z.one (Zonare Medical Systems, Mountain View, CA, USA) and a 3-10 MHz phased array (cardiac) transducer. An apical 5 chamber view of the heart will be obtained and the left-ventricular outflow tract visualised. A $3 \mathrm{~mm}$ pulsed-wave doppler sample volume will be gated at the level of the aortic valve and the resulting waveform recorded. The mean velocity-time integral (VTI) over three respiratory cycles will be recorded as the final VTI measurement. Left-ventricular outflow tract (LVOT) diameter will be measured from a parasternal long-axis (PLAX) view in systole. Stroke volume will be calculated as $\left(\pi r^{2}\right) \mathrm{x}$ velocity-time integral (VTI), and $\mathrm{CO}$ as stroke volume SV x HR. CI will be calculated as CO/BSA. Attempts to minimise the impact of inter and intra-observer variability on the study results include: blinding of the ultrasound assessor to the patient identity and timing of the study relative to the timing of fluid bolus administration; and assessment of intra and inter-rater reliability between the PI and the PI and blinded assessor, respectively, for all ultrasound-based assessments.

Extravascular lung water will be measured on lung ultrasound using a Zonare Z.one and an 5-14 MHz linear array probe set at a depth of $5 \mathrm{~cm}$. Eight views will be obtained and recorded as described in the Fluid Administration Limited by Lung Sonography (FALLS) protocol [40]. Lung ultrasound cine-loops will be scored in a blinded fashion for the presence of B-lines, confluent B-lines, or complete loss of lung aeration, and used to calculate a lung ultrasound score $[41,42]$.

Inferior vena cava collapsibility will be measured using a Zonare Z.one and a 1-4 MHz curved array transducer. A sub-costal long-axis view of the IVC will be obtained including the IVC-right atrial (RA) junction and confluence of the hepatic veins [43]. A cine-loop recording will 


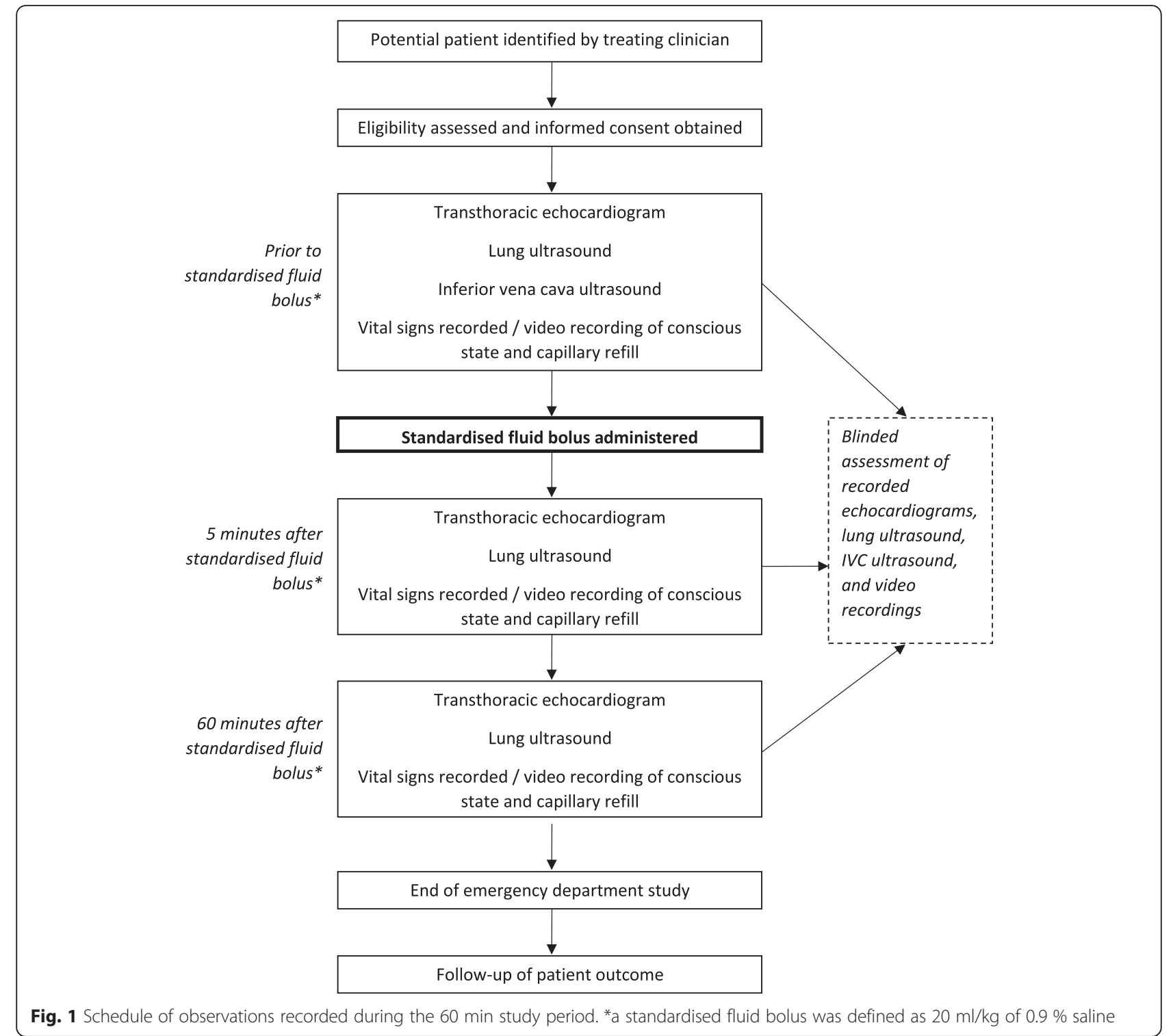

be taken over 3 respiratory cycles. IVC collapsibility will be measured at the level of the hepatic veins at endinspiration and end expiration [44]. IVC collapsibility will be calculated as: maximum - minimum diameter/ minimum diameter $\mathrm{x} 100$.

\section{Sample size, power, and statistical methods}

For a precision based sample size estimation based on the continuous outcome variable of change in cardiac output following standardised fluid bolus administration, the following assumptions have been made: a mean increase in cardiac output of $20 \%$ with a standard deviation of $10 \%$, a precision of the study tool (echocardiogram) of $+/-2 \%$. For a precision estimate of $5 \%$, a sample size of 50 would be required. This would also allow for regression analysis of several suspected confounding variables, including age, underlying illness aetiology (respiratory vs non-respiratory), and total volume of fluid previously administered.

\section{Limitations}

Ultrasound-based assessments are user-dependent. As such, they are prone to variability both within and between sonographers. Ultrasound-based assessment of EVLW and fluid responsiveness based on IVC collapsibility have been validated in adult patients but not in children. Age-based physiological differences in children may limit their utility in the study population. Some vital signs recorded (such as 
capillary refill, conscious state, and respiratory effort) are qualitative and prone to bias. Efforts to minimise the impact of potential bias on the study results include video recording of the patient at each time point and subsequent assessment blinded to the timing of the video recording relative to the timing of fluid bolus administration. At the time of assessment, enrolled patients are undifferentiated and are being treated empirically for sepsis. They may have an alternate final diagnosis. At the time of enrolment, patients are receiving multiple interventions, some of which are distressing and make ultrasound assessment challenging. Interventions other than fluid bolus administration (such as the use of non-invasive or invasive ventilation, or administration of inotropic or vaso-active therapies) may confound the study results. To minimise the effect of other therapies on study results, the study will focus only on the first hour of resuscitation. Any other interventions performed during the study period will be recorded. The study will only recruit patients when the PI is available in the ED. This limits the total number of patients recruited and the time of day when patients are recruited.

\section{Discussion}

Four major questions remain unanswered regarding fluid resuscitation for paediatric sepsis: what is the ideal fluid content, what is the ideal fluid volume, over what time period it should be administered, and what therapeutic targets should be used to titrate therapy. This study will help answer the questions of fluid volume and therapeutic targets. Current paediatric fluid resuscitation guidelines and planned trials are based on the concept of empiric fluid loading using vital signs as a marker of response to treatment. This is despite evidence that the host response to sepsis in children is highly variable and dynamic during the disease course [45-47], and that vital signs are a poor predictor of disease severity or response to treatment [48-51]. As such, using a "one size fits all" approach to fluid resuscitation seems counterintuitive. Alternatives include fluid responsiveness-based FRT. Data from randomised pilot studies in adults have shown fluid-responsiveness based resuscitation for sepsis allows more targeted fluid resuscitation (more aggressive in fluid responders, less aggressive in fluid non-responders), with an overall reduction in total fluid volume administered compared to standard treatment [52]. Additionally, the results of this study may suggest that routine assessment of EVLW allows for both the benefits and risks of fluid resuscitation to be included in the therapeutic equation for the individual patient. This approach has been described but not evaluated in randomised trials in adults [53].

This study may inform the design of future interventional studies in paediatric sepsis. The use of fluid-responsiveness based resuscitation limited by EVLW observed on lung ultrasound may allow targeted and individualised treatment
[54]. This may help avoid the harms from overzealous fluid resuscitation in some children. Such a strategy would need to have a significant impact on patient-centred outcomes for widespread use, due to its implications for resources, training, and infrastructure required in ED's caring for children.

\begin{abstract}
Abbreviations
$\mathrm{BP}$, blood pressure; $\mathrm{Cl}$, cardiac index; $\mathrm{CO}$, cardiac output; CVP, central venous pressure; ECMO, extracorporeal membrane oxygenation; $E D$, emergency department; EGDT, early goal-directed therapy; EVLW, extra-vascular lung water; FEAST, fluid expansion as supportive therapy; FRT, fluid resuscitation therapy; GCS, Glasgow Coma Scale; HR, heart rate; IVC, inferior vena cava; PI, principal investigator; $\mathrm{RCH}$, The Royal Children's Hospital; RR, respiratory rate; $\mathrm{SCvO2}$, central venous oxygen saturation; SIRS, systemic inflammatory response syndrome; SMD, septic myocardial dysfunction; SV, stroke volume
\end{abstract}

\section{Acknowledgements}

The authors would like to thank ED nursing and medical staff who have helped identify potential patients for recruitment and helped with study procedures. The PI would like to thank Dr Bennett Sheridan and Dr Adam O'brien for their help with interpretation of ultrasound images.

\section{Funding}

This study was supported in part by a Shields Research Entry Scholarship, The Royal Australasian College of Physicians, Sydney, Australia; by a National Health and Medical Research Council Centre of Research Excellence Grant for Pediatric Emergency Medicine (GNT1058560), Canberra, ACT, Australia; a Clinical Sciences Theme grant, Murdoch Children's Research Institute, Melbourne, Australia; and the Victorian Governments Infrastructure Support Program, Melbourne, Australia. FEB was supported in part by a grant from The Royal Children's Hospital Foundation.

Availability of data and materials

The dataset(s) supporting the conclusions of this article do not have ethics approval for public use.

\section{Authors' contributions}

All of the authors made substantial contributions to the concept and design of the study. EL drafted the original manuscript and all authors revised it critically. All authors have given final approval of the version to be published. All authors have agreed to be accountable for all aspects of the work ensuring that questions related to the accuracy or integrity of any part of the work are appropriately investigated and resolved.

\section{Competing interests}

The authors declare that they have no competing interests.

\section{Ethics approval and consent to participate}

The study has ethics approval at the study hospital (Human Research Ethics Committee 33169A). All parents/guardians (and patients where appropriate) will be provided with verbal and written information about the study and

written informed consent will be obtained prior to enrolment into the study.

\section{Author details}

'Department of Emergency Medicine, The Royal Children's Hospital, 50 Flemington Road, 3052 Parkville, VIC, Australia. ${ }^{2}$ Murdoch Children's Research Institute, 50 Flemington Road, Parkville, Australia. ${ }^{3}$ Department of Paediatrics, Faculty of Medicine, Dentistry, and Health Sciences, University of Melbourne, Victoria, Australia. ${ }^{4}$ Paediatric Intensive Care Unit, The Royal Children's Hospital, 50 Flemington Road, Parkville, VIC, Australia.

Received: 25 December 2015 Accepted: 9 July 2016

Published online: 15 July 2016

\section{References}

1. Black RE, Cousens S, Johnson HL, Lawn JE, Rudan I, Bassani DG, Jha P, Campbell H, Walker CF, Cibulskis R, et al. Global, regional, and national causes of child mortality in 2008: a systematic analysis. Lancet. 2010; 375(9730):1969-87. 
2. Watson RS, Carcillo JA. Scope and epidemiology of pediatric sepsis. Pediatr Crit Care Med. 2005;6(3 Suppl):S3-5.

3. Jaramillo-Bustamante JC, Marín-Agudelo A, Fernández-Laverde M, Bareño-Silva J. Epidemiology of sepsis in pediatric intensive care units: First Colombian Multicenter Study*. Pediatr Crit Care Med. 2012;13(5):501-8. doi:10.1097/PCC. 1090b1013e31823c31980f.

4. Brierley J, Carcillo JA, Choong K, Cornell T, Decaen A, Deymann A, Doctor A, Davis A, Duff J, Dugas MA, et al. Clinical practice parameters for hemodynamic support of pediatric and neonatal septic shock: 2007 update from the American College of Critical Care Medicine. Crit Care Med. 2009; 37(2):666-88.

5. Maconochie IK, de Caen AR, Aickin R, Atkins DL, Biarent D, Guerguerian AM, Kleinman ME, Kloeck DA, Meaney PA, Nadkarni VM, et al. Part 6: Pediatric basic life support and pediatric advanced life support: 2015 International Consensus on Cardiopulmonary Resuscitation and Emergency Cardiovascular Care Science with Treatment Recommendations. Resuscitation. 2015;95:e147-68.

6. Cosnett JE. The origins of intravenous fluid therapy. Lancet. 1989; (8641):768-71.

7. Myburgh JA, Mythen MG. Resuscitation fluids. N Engl J Med. 2013;369(25): 2462-3.

8. Rivers E, Nguyen B, Havstad S, Ressler J, Muzzin A, Knoblich B, Peterson E, Tomlanovich M. Early Goal-Directed Therapy in the Treatment of Severe Sepsis and Septic Shock. N Engl J Med. 2001;345(19):1368-77.

9. Boyd JH, Forbes J, Nakada TA, Walley KR, Russell JA. Fluid resuscitation in septic shock: a positive fluid balance and elevated central venous pressure are associated with increased mortality. Crit Care Med. 2011;39(2):259-65.

10. Arikan AA, Zappitelli M, Goldstein SL, Naipaul A, Jefferson LS, Loftis LL. Fluid overload is associated with impaired oxygenation and morbidity in critically ill children. Pediatr Crit Care Med. 2012;13(3):253-8.

11. Foland JA, Fortenberry JD, Warshaw BL, Pettignano R, Merritt RK, Heard ML, Rogers K, Reid C, Tanner AJ, Easley KA. Fluid overload before continuous hemofiltration and survival in critically ill children: a retrospective analysis. Crit Care Med. 2004;32(8):1771-6.

12. Rosenberg AL, Dechert RE, Park PK, Bartlett RH. Review of a large clinical series: association of cumulative fluid balance on outcome in acute lung injury: a retrospective review of the ARDSnet tidal volume study cohort. J Intensive Care Med. 2009;24(1):35-46.

13. Cordemans C, De Laet I, Van Regenmortel N, Schoonheydt K, Dits H, Huber W, Malbrain ML. Fluid management in critically ill patients: the role of extravascular lung water, abdominal hypertension, capillary leak, and fluid balance. Ann Intensive Care. 2012;2(Suppl 1 Diagnosis and management of intra-abdominal hyperten):S1.

14. Micek ST, McEvoy C, McKenzie M, Hampton N, Doherty JA, Kollef MH. Fluid balance and cardiac function in septic shock as predictors of hospital mortality. Crit Care. 2013;17(5):R246.

15. Selewski DT, Cornell TT, Lombel RM, Blatt NB, Han YY, Mottes T, Kommareddi M, Kershaw DB, Shanley TP, Heung M. Weight-based determination of fluid overload status and mortality in pediatric intensive care unit patients requiring continuous renal replacement therapy. Intensive Care Med. 2011;37(7):1166-73.

16. Sutherland SM, Zappitelli M, Alexander SR, Chua AN, Brophy PD, Bunchman TE, Hackbarth R, Somers MJ, Baum M, Symons JM, et al. Fluid overload and mortality in children receiving continuous renal replacement therapy: the prospective pediatric continuous renal replacement therapy registry. Am J Kidney Dis. 2010:55(2):316-25.

17. Peake SL, Delaney A, Bailey M, Bellomo R, Cameron PA, Cooper DJ, Higgins AM, Holdgate A, Howe BD, Webb SA, et al. Goal-directed resuscitation for patients with early septic shock. N Engl J Med. 2014;371(16):1496-506.

18. Yealy DM, Kellum JA, Huang DT, Barnato AE, Weissfeld LA, Pike F, Terndrup T, Wang HE, Hou PC, LoVecchio F, et al. A randomized trial of protocol-based care for early septic shock. N Engl J Med. 2014;370(18):1683-93.

19. Mouncey PR, Osborn TM, Power GS, Harrison DA, Sadique MZ, Grieve RD, Jahan R, Harvey SE, Bell D, Bion JF, et al. Trial of Early, Goal-Directed Resuscitation for Septic Shock. N Engl J Med. 2015.

20. Maitland K, Kiguli S, Opoka RO, Engoru C, Olupot-Olupot P, Akech SO, Nyeko R, Mtove G, Reyburn $\mathrm{H}$, Lang $\mathrm{T}$, et al. Mortality after fluid bolus in African children with severe infection. N Engl J Med. 2011;364(26):2483-95.

21. Malbrain ML, Marik PE, Witters I, Cordemans C, Kirkpatrick AW, Roberts DJ, Van Regenmortel N. Fluid overload, de-resuscitation, and outcomes in critically ill or injured patients: a systematic review with suggestions for clinical practice. Anaesthesiology intensive therapy. 2014;46(5):361-80.
22. Henderson WR, Griesdale DE, Walley KR, Sheel AW. Clinical review: Guytonthe role of mean circulatory filling pressure and right atrial pressure in controlling cardiac output. Crit Care. 2010;14(6):243.

23. Applegate RJ, Johnston WE, Vinten-Johansen J, Klopfenstein HS, Little WC. Restraining effect of intact pericardium during acute volume loading. Am J Physiol. 1992;262(6 Pt 2):H1725-33.

24. Funk DJ, Jacobsohn E, Kumar A. The role of venous return in critical illness and shock-part I: physiology. Crit Care Med. 2013;41(1):255-62.

25. Vellinga NA, Ince C, Boerma EC. Elevated central venous pressure is associated with impairment of microcirculatory blood flow in sepsis: a hypothesis generating post hoc analysis. BMC Anesthesiol. 2013;13:17.

26. Reitsma S, Slaaf DW, Vink H, van Zandvoort MA, oude Egbrink MG. The endothelial glycocalyx: composition, functions, and visualization. Pflugers Arch. 2007;454(3):345-59.

27. Woodcock TE, Woodcock TM. Revised Starling equation and the glycocalyx model of transvascular fluid exchange: an improved paradigm for prescribing intravenous fluid therapy. Br J Anaesth. 2012;108(3):384-94.

28. Levick JR, Michel CC. Microvascular fluid exchange and the revised Starling principle. Cardiovasc Res. 2010;87(2):198-210.

29. Ait-Oufella H, Maury E, Lehoux S, Guidet B, Offenstadt G. The endothelium: physiological functions and role in microcirculatory failure during severe sepsis. Intensive Care Med. 2010;36(8):1286-98.

30. Trzeciak S, Rivers EP. Clinical manifestations of disordered microcirculatory perfusion in severe sepsis. Crit Care. 2005;9 Suppl 4:S20-6.

31. Prowle JR, Echeverri JE, Ligabo EV, Ronco C, Bellomo R. Fluid balance and acute kidney injury. Nat Rev Nephrol. 2010;6(2):107-15.

32. Marik PE. latrogenic salt water drowning and the hazards of a high central venous pressure. Ann Intensive Care. 2014;4:21.

33. Nunes TS, Ladeira RT, Bafi AT, de Azevedo LC, Machado FR, Freitas FG. Duration of hemodynamic effects of crystalloids in patients with circulatory shock after initial resuscitation. Ann Intensive Care. 2014:4:25.

34. Caltabeloti F, Monsel A, Arbelot C, Brisson H, Lu Q, Gu WJ, Zhou GJ, Auler JO, Rouby $\mathrm{JJ}$. Early fluid loading in acute respiratory distress syndrome with septic shock deteriorates lung aeration without impairing arterial oxygenation: a lung ultrasound observational study. Crit Care. 2014;18(3):R91.

35. Marik PE, Monnet X, Teboul JL. Hemodynamic parameters to guide fluid therapy. Ann Intensive Care. 2011;1 (1):1.

36. Feihl F, Broccard AF. Interactions between respiration and systemic hemodynamics. Part I: basic concepts. Intensive Care Med. 2009;35(1):45-54.

37. Muller L, Bobbia X, Toumi M, Louart G, Molinari N, Ragonnet B, Quintard H, Leone M, Zoric L, Lefrant JY. Respiratory variations of inferior vena cava diameter to predict fluid responsiveness in spontaneously breathing patients with acute circulatory failure: need for a cautious use. Crit Care. 2012;16(5):R188.

38. Goldstein B, Giroir B, Randolph A. International pediatric sepsis consensus conference: definitions for sepsis and organ dysfunction in pediatrics. Pediatr Crit Care Med. 2005;6(1):2-8

39. Silverman WA, Andersen DH. A controlled clinical trial of effects of water mist on obstructive respiratory signs, death rate and necropsy findings among premature infants. Pediatrics. 1956;17(1):1-10.

40. Lichtenstein D, Karakitsos D. Integrating lung ultrasound in the hemodynamic evaluation of acute circulatory failure (the fluid administration limited by lung sonography protocol). J Crit Care. 2012:27(5):533.e511-539.

41. Bouhemad B, Zhang M, Lu Q, Rouby JJ. Clinical review: Bedside lung ultrasound in critical care practice. Crit Care. 2007:11(1):205.

42. Soummer A, Perbet S, Brisson H, Arbelot C, Constantin JM, Lu Q, Rouby JJ. Ultrasound assessment of lung aeration loss during a successful weaning trial predicts postextubation distress*. Crit Care Med. 2012;40(7):2064-72.

43. Kircher BJ, Himelman RB, Schiller NB. Noninvasive estimation of right atrial pressure from the inspiratory collapse of the inferior vena cava. Am J Cardiol. 1990;66(4):493-6.

44. Feissel M, Michard F, Faller JP, Teboul JL. The respiratory variation in inferior vena cava diameter as a guide to fluid therapy. Intensive Care Med. 2004; 30(9):1834-7.

45. Deep A, Goonasekera CD, Wang Y, Brierley J. Evolution of haemodynamics and outcome of fluid-refractory septic shock in children. Intensive Care Med. 2013;39(9):1602-9.

46. Ranjit S, Aram G, Kissoon N, Ali MK, Natraj R, Shresti S, Jayakumar I, Gandhi D. Multimodal monitoring for hemodynamic categorization and management of pediatric septic shock: a pilot observational study*. Pediatr Crit Care Med. 2014; 15(1):e17-26. 
47. Brierley J, Peters MJ. Distinct hemodynamic patterns of septic shock at presentation to pediatric intensive care. Pediatrics. 2008;122(4):752-9.

48. Scott HF, Donoghue AJ, Gaieski DF, Marchese RF, Mistry RD. Effectiveness of physical exam signs for early detection of critical illness in pediatric systemic inflammatory response syndrome. BMC Emerg Med. 2014;14:24.

49. Saugel B, Ringmaier S, Holzapfel K, Schuster T, Phillip V, Schmid RM, Huber W. Physical examination, central venous pressure, and chest radiography for the prediction of transpulmonary thermodilution-derived hemodynamic parameters in critically ill patients: a prospective trial. J Crit Care. 2011;26(4): 402-10.

50. Nowak RM, Sen A, Garcia AJ, Wilkie H, Yang JJ, Nowak MR, Moyer ML. The inability of emergency physicians to adequately clinically estimate the underlying hemodynamic profiles of acutely ill patients. Am J Emerg Med. 2012;30(6):954-60.

51. Glassford NJ, Eastwood GM, Bellomo R. Physiological changes after fluid bolus therapy in sepsis: a systematic review of contemporary data. Crit Care. 2014;18(6):696.

52. Richard J-C, Bayle F, Bourdin G, Leray V, Debord S, Delannoy B, Stoian AC, Wallet $F$, Yonis $H$, Guerin $C$. Preload dependence indices to titrate volume expansion during septic shock: a randomized controlled trial. Crit Care. 2015;19(1):5.

53. Lichtenstein D. FALLS-protocol: lung ultrasound in hemodynamic assessment of shock. Heart, lung and vessels. 2013;5(3):142-7.

54. Marik P, Bellomo R. A rational approach to fluid therapy in sepsis. Br J Anaesth. 2015

55. Daymont C, Bonafide CP, Brady PW. Heart rates in hospitalized children by age and body temperature. Pediatrics. 2015;135(5):e1173-81.

\section{Submit your next manuscript to BioMed Central and we will help you at every step:}

- We accept pre-submission inquiries

- Our selector tool helps you to find the most relevant journal

- We provide round the clock customer support

- Convenient online submission

- Thorough peer review

- Inclusion in PubMed and all major indexing services

- Maximum visibility for your research

Submit your manuscript at www.biomedcentral.com/submit

) Biomed Central 